TRANSACTIONS OF THE

AMERICAN MATHEMATICAL SOCIETY

Volume 350, Number 8, August 1998, Pages 3393-3405

S 0002-9947(98)02317-4

\title{
FILLING-INVARIANTS AT INFINITY FOR MANIFOLDS OF NONPOSITIVE CURVATURE
}

\author{
NOEL BRADY AND BENSON FARB
}

\begin{abstract}
In this paper we construct and study isoperimetric functions at infinity for Hadamard manifolds. These quasi-isometry invariants give a measure of the spread of geodesics in such a manifold.
\end{abstract}

\section{INTRODUCTION}

Homological invariants "at infinity" and (coarse) isoperimetric inequalities are basic tools in the study of large-scale geometry (see, e.g., [Gr, BW]). The purpose of this paper is to combine these two ideas to construct a family $\operatorname{div}_{k}\left(X^{n}\right), 0 \leq k \leq$ $n-2$, of geometric invariants for complete, simply-connected manifolds $X^{n}$ with nonpositive sectional curvatures. The $\operatorname{div}_{k}\left(X^{n}\right)$ are meant to give a finer measure of the spread of geodesics in $X^{n}$; in fact the 0 -th invariant $\operatorname{div}_{0}\left(X^{n}\right)$ is the well-known "rate of divergence of geodesics" in the Riemannian manifold $X^{n}$.

The definition of $\operatorname{div}_{k}\left(X^{n}\right)$ goes roughly as follows (see Section 1 for the precise definitions): Find the minimum volume of a ball $B^{k+1}$ needed to fill a sphere $S^{k}$, where $S^{k}$ sits on the $(n-1)$-dimensional sphere $S(r)$ of radius $r$ in $X^{n}$, and the filling ball $B^{k+1}$ is required to lie outside the $n$-dimensional open ball $B(r)^{\circ}$ in $X^{n}$. Then $\operatorname{div}_{k}\left(X^{n}\right)$ measures the growth of this volume as $r \rightarrow \infty$; hence $\operatorname{div}_{k}\left(X^{n}\right)$ is in some sense a $k$-dimensional isoperimetric function at infinity.

The $\operatorname{div}_{k}\left(X^{n}\right)$ are quasi-isometry invariants of $X^{n}$. The fundamental group $\pi_{1}\left(M^{n}\right)$ (endowed with the word metric) of a compact Riemannian manifold is quasi-isometric to the universal cover $\widetilde{M^{n}}$; hence the $\operatorname{div}_{k}\left(\widetilde{M^{n}}\right)$ give quasi-isometry invariants for fundamental groups of closed, nonpositively curved manifolds $M^{n}$.

This was our intial motivation for studying the $\operatorname{div}_{k}\left(X^{n}\right)$ (see below for an application). But we also feel that the invariants $\operatorname{div}_{k}\left(X^{n}\right)$ should be viewed in the same way as the standard isoperimetric inequalities (for manifolds or for groups): as basic geometric quantities to be computed. In particular, computations of $\operatorname{div}_{k}(X)$ provide a good test of one's understanding of the geometry of $X$.

The contents of this paper are as follows: In Section 1, $\operatorname{div}_{k}\left(X^{n}\right)$ is defined and shown to be a quasi-isometry invariant. The core of this paper (Sections $2,3,4)$ describes three geometric techniques for computing $\operatorname{div}_{k}\left(X^{n}\right)$ for some basic examples. Section 4 also explores some surprising quasi-isometric embeddings of hyperbolic spaces and solvable Lie groups into products of hyperbolic spaces.

Received by the editors April 18, 1995 and, in revised form, October 25, 1996.

1991 Mathematics Subject Classification. Primary 53C23, 20F32, 57M07.

The second author is supported in part by an N.S.F. Postdoctoral Fellowship.

(C)1998 American Mathematical Society 
We would like to express our gratitude to K. Fujiwara, C. Pugh, and A. Wilkinson for helpful discussions, and to G. Kuperburg for doing the first two figures. We would also like to thank the referee for making many useful comments and corrections.

\section{Definitions and Quasi-Isometry Invariance}

Let $X^{n}$ be an Hadamard manifold; that is, a complete, simply connected manifold all of whose sectional curvatures are nonpositive. Let $S(r), B(r)$ and $B(r)^{\circ}$ denote respectively the sphere, ball and open ball of radius $r$ about a fixed basepoint $x_{0}$ of $X^{n}$, and let $C(r)=X^{n} \backslash B(r)^{\circ}$. Note that $C(r)$ deformation retracts onto the sphere $S(r)$; hence any continuous map $f: S^{k} \rightarrow S(r)$ admits a continuous extension, or filling $\widehat{f}: B^{k+1} \rightarrow C(r)$, for any integer $0 \leq k \leq n-2$.

We shall be considering lipschitz maps to the manifold $X^{n}$. By the Whitney Extension Theorem, we know that if $f$ as above is lipschitz, then the extension $\widehat{f}$ of $f$ can be chosen to be lipschitz (with the same lipschitz constant). By Rademacher's Theorem, lipschitz maps are differentiable almost everywhere, enabling one to define the $k$-volume of $f: S^{k} \rightarrow X^{n}$ and the $(k+1)$-volume of $\widehat{f}: B^{k+1} \rightarrow X^{n}$, where $S^{k}$ and $B^{k+1}$ denote the unit sphere and ball in euclidean space $\mathbf{R}^{n}$. More precisely, if the derivative $D_{x} f$ exists at a point $x \in S^{k}$, it sends an orthonormal basis at $x$ to a $k$-tuple of vectors in $T_{f(x)}\left(X^{n}\right)$. We can compute the $k$-volume of the parallelopiped spanned by this $k$-tuple using the metric on $X^{n}$. This defines a function $V(x)$ almost everywhere on $S^{k}$, and we can then define the $k$-volume of $f$, denoted $\operatorname{vol}_{k}(f)$, to be the integral of $V$ over $S^{k}$. This integral exists because $V(x)$ is a bounded measurable function defined almost everywhere on $S^{k}$, as $\left\|D_{x} f\right\|$ is bounded by the lipschitz constant of $f$.

We are now ready to define the invariant $\operatorname{div}_{k}\left(X^{n}\right)$ for a fixed integer $0 \leq k \leq$ $n-2$. Although the concept of $\operatorname{div}_{k}$ is quite simple, the precise definition of $\operatorname{div}_{k}$ needs to be somewhat technical in order to make it manifestly a quasi-isometry invariant (see Theorem 1.1). For example, the constants $A$ and $\rho$ below are needed to deal with stretching effects of quasi-isometries on spheres. This method of getting a quasi-isometry invariant is a variation of a trick introduced in [Ge1].

Let $A>0$ and $0<\rho \leq 1$ be given. For $r>0$, we define a map $f: S^{k} \rightarrow S(r)$ to be $A$-admissible if

- $f$ is lipschitz, and

- $\operatorname{vol}_{k}(f) \leq A r^{k}$,

and say that the extension $\widehat{f}$ of $f$ is $\rho$-admissible if

- $\widehat{f}$ is lipschitz, and

- $\widehat{f}\left(B^{k+1}\right) \subset C(\rho r)$.

In other words, the only admissible fillings are those which lie outside the open ball $B^{\circ}(\rho r)$ in $X^{n}$. See Figure 1. Now define

$$
\delta_{\rho, A}^{k}(r)=\sup _{f} \inf _{\widehat{f}} \operatorname{vol}_{k+1}(\widehat{f}),
$$

where the supremun is taken over all $A$-admissible maps $f$ and the infimum is taken over all $\rho$-admissible fillings $\widehat{f}$ of $f$.

We need the polynomial bound on $\operatorname{vol}_{k}(f)$ in the definition above since we want to distinguish between cases when the growth of the $\delta_{\rho, A}^{k}(r)$ is polynomial versus 


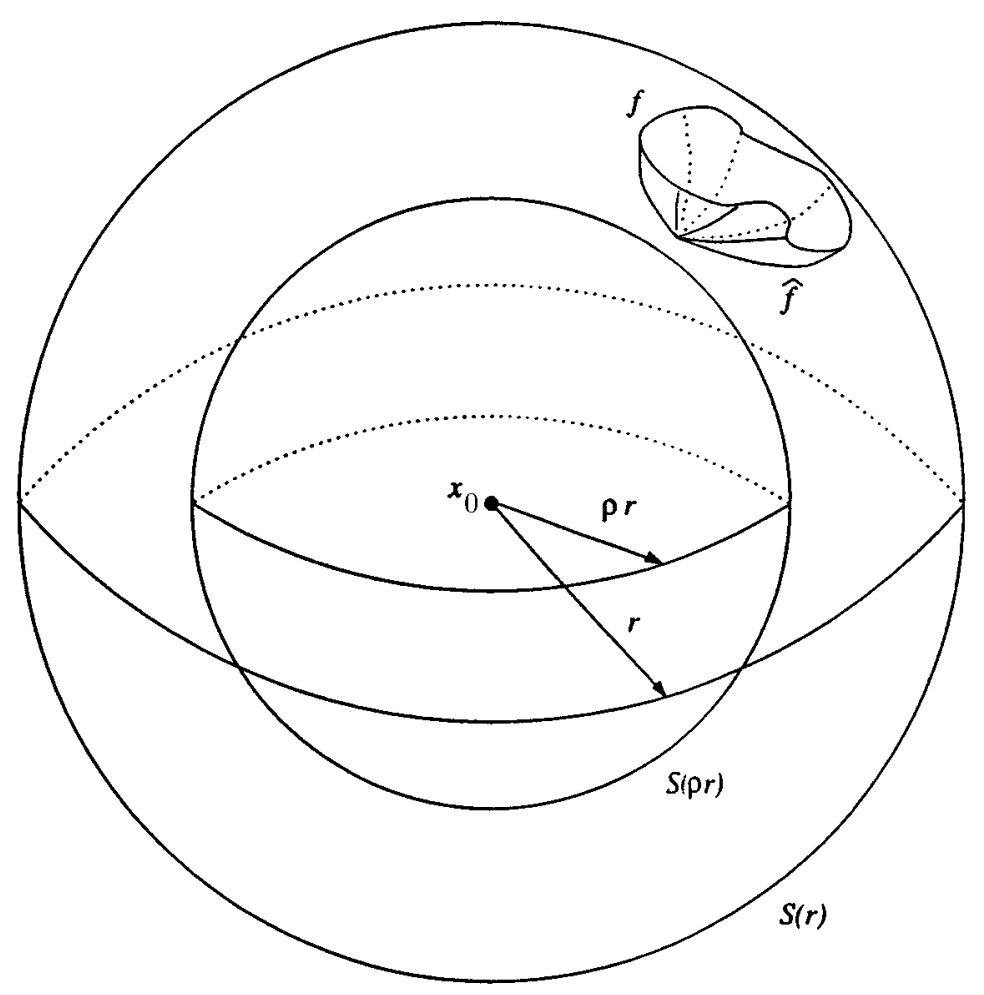

Figure 1. An admissible filling $\hat{f}$.

exponential. Without this polynomial bound, one could construct exponential $k$ volume maps $f$ requiring exponential volume extensions, so that the $\delta_{\rho, A}^{k}(r)$ would always be at least exponential.

Definition. The $k$-th divergence of $X$, denoted $\operatorname{div}_{k}(X)$, is defined to be the two parameter family of functions

$$
\operatorname{div}_{k}\left(X^{n}\right)=\left\{\delta_{\rho, A}^{k}(r): 0<\rho \leq 1, A>0\right\} .
$$

Remark. We note that the function $\delta_{\rho, A}^{k}(r)$, as a sup of an inf, may not be realized by an actual filling, though of course there are (admissible) fillings arbitrarily close to realizing this function. We will ignore this distinction in what follows, as we are only interested in the growth of $\delta_{\rho, A}^{k}(r)$.

In this paper we shall only be concerned with distinguishing between polynomial and exponential functions. Hence the following equivalence relation: given functions $f, g: \mathbf{R}^{+} \rightarrow \mathbf{R}^{+}$, we write $f \preceq g$ if there exist constants $a, b, c>0$ and an integer $s \geq 0$ such that $f(x) \leq a g(b x)+c x^{s}$ for all sufficiently large $x$. Now write $f \sim g$ if both $f \preceq g$ and $g \preceq f$. This defines an equivalence relation on the class of functions from $\mathbf{R}^{+}$to $\mathbf{R}^{+}$, and it makes sense to call the equivalence classes polynomial, exponential, super-exponential, etc.

Definition. We say that $\operatorname{div}_{k} \preceq \operatorname{div}_{k}^{\prime}$ if there exist $0<\rho_{0}, \rho_{0}^{\prime} \leq 1$ and $A_{0}, A_{0}^{\prime}>0$ such that for every pair $(\rho, A)$ with $\rho<\rho_{0}$ and $A>A_{0}$ there exist $\rho^{\prime}<\rho_{0}^{\prime}$ and 
$A^{\prime}>A_{0}^{\prime}$ with $\delta_{\rho, A}^{k} \preceq{\delta^{\prime}}_{\rho^{\prime}, A^{\prime}}^{k}$. Now define $\operatorname{div}_{k} \sim \operatorname{div}_{k}^{\prime}$ if we have both $\operatorname{div}_{k} \preceq \operatorname{div}_{k}^{\prime}$ and $\operatorname{div}_{k}^{\prime} \preceq \operatorname{div}_{k}$.

We say that $\operatorname{div}_{k}$ is polynomial or exponential if the following holds: there exist $0<\rho_{0} \leq 1$ and $0<A_{0}$ such that $\delta_{\rho, A}(r) \preceq r^{d}$ (for some integer $d>0$ ) or $\delta_{\rho, A}(r) \succeq e^{r}$ respectively, for all $\rho<\rho_{0}$ and $A>A_{0}$.

We are now ready to prove that the invariants $\operatorname{div}_{k}\left(X^{n}\right)$ are actually quasiisometry invariants of $X^{n}$, sometimes called geometric invariants. Recall that a quasi-isometry is basically a coarse bilipschitz map; these are the appropriate maps to study when one is interested in large-scale geometric properties of a space, or in geometric properties of the fundamental group of a compact Riemannian manifold (see, e.g., $[\mathrm{Gr}]$ ). More precisely, we recall the following:

Definition. Let $X$ and $Y$ be metric spaces. A quasi-isometry is a pair of maps $f: X \longrightarrow Y, g: Y \longrightarrow X$ such that, for some constants $K, \epsilon, C>0$,

$$
\begin{array}{ll}
d_{Y}\left(f\left(x_{1}\right), f\left(x_{2}\right)\right) \leq K d_{X}\left(x_{1}, x_{2}\right)+\epsilon, & d_{X}\left(g \circ f\left(x_{1}\right), x_{1}\right) \leq C, \\
d_{X}\left(g\left(y_{1}\right), g\left(y_{2}\right)\right) \leq K d_{Y}\left(y_{1}, y_{2}\right)+\epsilon, & d_{Y}\left(f \circ g\left(y_{1}\right), y_{1}\right) \leq C,
\end{array}
$$

for all $x_{1}, x_{2} \in X, y_{1}, y_{2} \in Y$. Note that neither $f$ nor $g$ need be continuous. If such maps exist, $X$ and $Y$ are said to be quasi-isometric; the map $f$ is called a $K$ quasi-isometry. A quasi-isometric embedding is defined similarly. A basic example to keep in mind is that the fundamental group $\pi_{1}(M)$ (endowed with the word metric) of a compact Riemannian manifold $M$ is quasi-isometric to the universal cover $\widetilde{M}$ of $M$.

Theorem 1.1 ( $\operatorname{div}_{k}$ is a quasi-isometry invariant). Let $X$ and $Y$ be Hadamard $n$ manifolds. If $X$ and $Y$ are quasi-isometric, then $\operatorname{div}_{k}(X) \sim \operatorname{div}_{k}(Y)$ for all $0 \leq$ $k \leq n-2$. In particular, $\operatorname{div}_{k}$ gives a quasi-isometry invariant for fundamental groups of closed, nonpositively curved manifolds.

Proof of Theorem 1.1. Let $F:\left(X, x_{0}\right) \rightarrow\left(Y, y_{0}\right)$ and $G:\left(Y, y_{0}\right) \rightarrow\left(X, x_{0}\right)$ be $L$ lipschitz maps between two based Hadamard manifolds which are determined by a quasi-isometry between $X$ and $Y$ (see Appendix A for existence of lipschitz quasi-

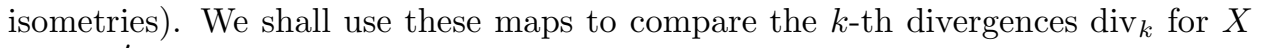
and $\operatorname{div}_{k}^{\prime}$ for $Y$.

Given an $A$-admissible map $f: S^{k} \rightarrow S_{X}(r)$, we can compose with $F$ to get a lipschitz map $F \circ f: S^{k} \rightarrow Y$ with $(F \circ f)\left(S^{k}\right) \subset B_{Y}(L r) \cap C_{Y}(r / L)$. Note that $\operatorname{vol}_{k}(F \circ f) \leq A(L r)^{k}$. Radial projection onto $S_{Y}(r / L)$ defines a volume nonincreasing lipschitz map (lipschitz constant 1) $\pi: C_{Y}(r / L) \rightarrow S_{Y}(r / L)$, and so the composition $(\pi \circ F \circ f): S^{k} \rightarrow S_{Y}(r / L)$ is $A L^{2 k}$-admissible (see Figure 2 ). There is an admissible filling of this map with $(k+1)$-volume bounded by $\delta_{\rho, A / L^{2 k}}^{\prime k}(r / L)$.

We obtain a lipschitz extension of $F \circ f$ as follows: on the radius one-half ball in $B^{k+1}$ take the map $(\pi \widehat{\circ F \circ} f) \circ d_{2}$, where $d_{2}$ denotes the dilation taking the radius one-half ball $\frac{1}{2} B^{k+1}$ onto $B^{k+1}$, and on the remaining annular region just interpolate between the maps $F \circ f$ and $(\pi \widehat{\circ F \circ} f) \circ d_{2}$ (sending radial geodesics in $B^{k+1}$ to geodesic segments between image points in $Y$ ). Note that the $(k+1)$ volume of this map is bounded by $\delta_{\rho, A L^{2 k}}^{\prime}(r / L)$ plus a polynomial of degree $k+1$ in $r$. This polynomial bounds the $(k+1)$-volume of the annular region, and is the reason we use the equivalence relation among functions defined above.

Now postcomposition with $G$ yields a lipschitz map from $B^{k+1}$ to $X$ which lies outside the $\rho r / l^{2}$-ball about $x_{0}$, and the restriction of this map to $S^{k}$ is a constant 


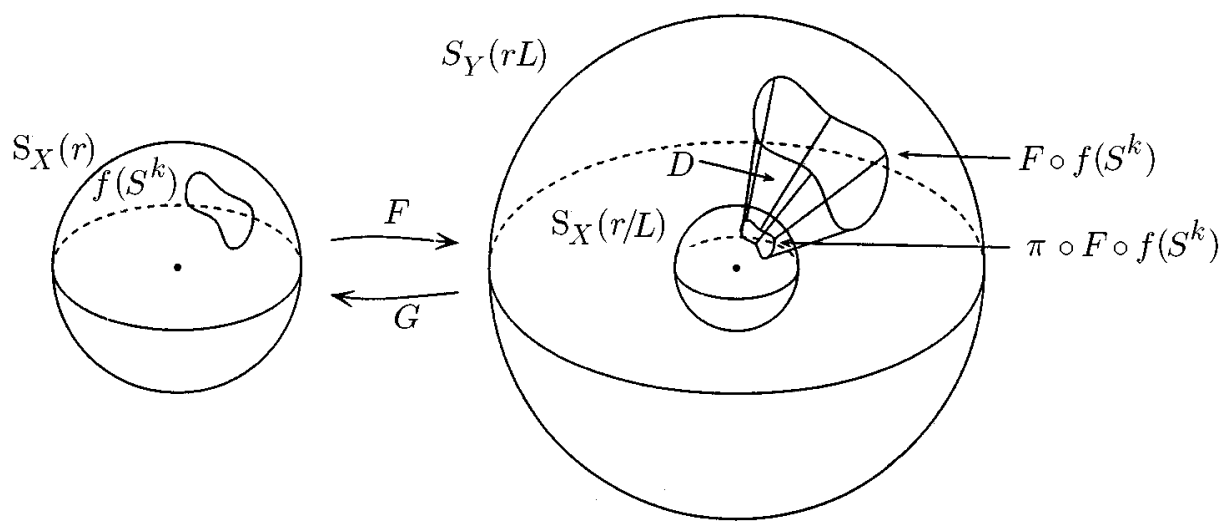

Figure 2. This figure illustrates part of the proof of Theorem 1.1.

distance (pointwise) away from the original map $f: S^{k} \rightarrow X$, as $G \circ F$ is a constant distance away from the identity map $1_{X}$. It is easy to see that one can interpolate between these maps to obtain a lipschitz map $\widehat{f}: B^{k+1} \rightarrow X$ which is a $\rho / L^{2}$ admissible filling of $f$. Note that

$$
\operatorname{vol}_{k+1}(\widehat{f}) \leq L^{k+1} \delta_{\rho, A L^{2 k}}^{\prime}(r / L)+p(r),
$$

where $p(r)$ is a polynomial of degree $k+1$ in $r$, and so

$$
\delta_{\rho / L^{2}, A}(r) \preceq \delta_{\rho, A L^{2 k}}^{\prime}(r) .
$$

Thus, $\operatorname{div}_{k} \preceq \operatorname{div}_{k}^{\prime}$. Similarly, $\operatorname{div}_{k}^{\prime} \preceq \operatorname{div}_{k}$, and so $\operatorname{div}_{k}$ is a true quasi-isometry invariant.

\section{Suspending Hard-TO-Fill Spheres}

In this section we show how to suspend hard-to-fill spheres in $X$ to hard-to-fill spheres in $X \times \mathbf{R}$. This provides a lower bound for the $(k+1)$ st divergence of $X \times \mathbf{R}$ in terms of the $k$ th divergence for $X$.

Theorem 2.1 (suspending hard-to-fill spheres). Let $X^{n}$ be an Hadamard manifold. Then

$$
\operatorname{div}_{k+1}\left(X^{n} \times \mathbf{R}\right) \geq \operatorname{div}_{k}\left(X^{n}\right) \quad \text { for any } 0 \leq k \leq n-2 .
$$

Theorem 2.1 shows, for example, that

$$
\operatorname{div}_{2}\left(\mathbf{H}^{2} \times \mathbf{R}^{2}\right) \geq \operatorname{div}_{1}\left(\mathbf{H}^{2} \times \mathbf{R}\right) \geq \operatorname{div}_{0}\left(\mathbf{H}^{2}\right) \sim \exp .
$$

Proof of Theorem 2.1. Denote $X^{n}$ simply by $X$, and let $S(r)$ be the sphere of radius $r$ in $X \times \mathbf{R}$. Since $X=X \times\{0\}$ is totally geodesic in $X \times \mathbf{R}$, the intersection $S^{\prime}(r)=S(r) \cap X$ is the sphere of radius $r$ in $X$. Choose an admissible map $f: S^{k} \rightarrow S^{\prime}(r)$ which realizes $\operatorname{div}_{k}(X)$. We now define a map

$$
\Sigma(f): \Sigma\left(S^{k}\right)=S^{k+1} \longrightarrow S(r),
$$

where $\Sigma\left(S^{k}\right)$ denotes the suspension $S^{k} \times[0,1] /\left(\left(S^{k} \times\{0\}\right) \cup\left(S^{k} \times\{1\}\right)\right)$ of $S^{k}$. Geometrically, define the map $\Sigma(f)$ as follows: for $p \in S^{k}$, let $F_{p}$ be the 2-flat which is the product of the infinite geodesic in $X$ passing through $x_{0}$ and $f(p)$ 


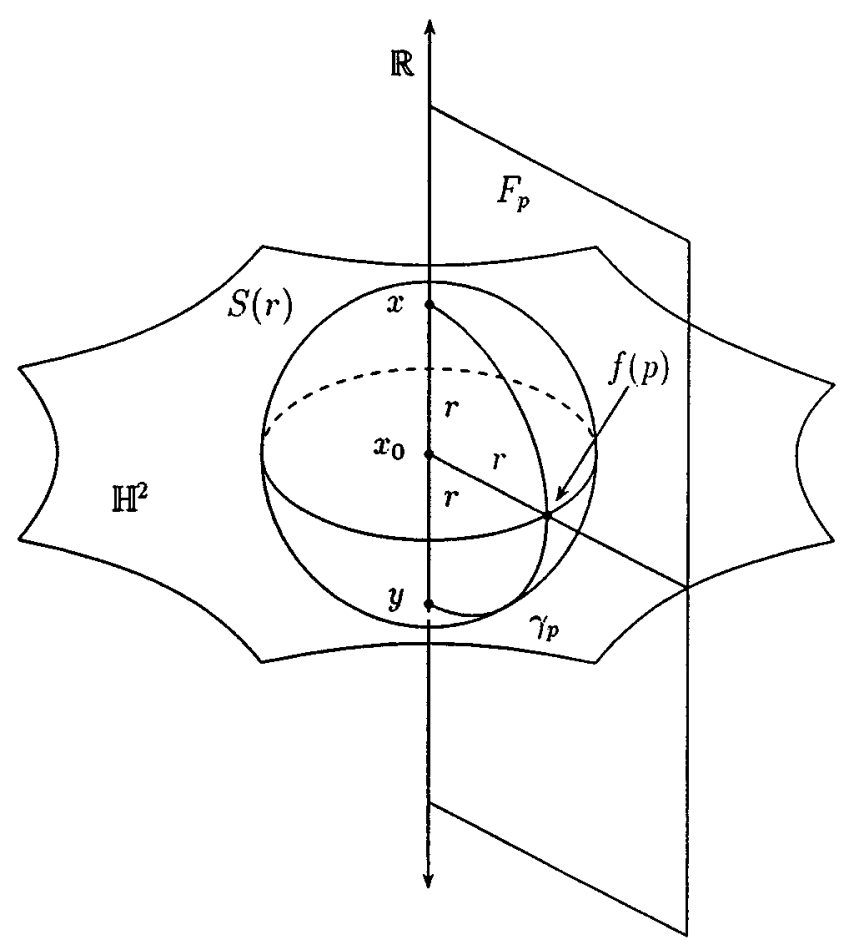

Figure 3. The $\operatorname{arc} \gamma_{p}$ is used to define $\Sigma(f)$ at the point $\{p\} \times[0,1]$ of the suspension $\Sigma\left(S^{k}\right)=S^{k+1}$. This figure illustrates the case $X=\mathbf{H}^{2}, k=1$.

and the infinite geodesic $\left\{x_{0}\right\} \times \mathbf{R}$. We think of each $F_{p}$ based at the "origin" $\left(x_{0}, 0\right) \in X \times \mathbf{R}$, and note that the points $x=\left(x_{0}, r\right)$ and $y=\left(x_{0},-r\right)$ of $S(r)$ are contained in each $F_{p}$. Let $\gamma_{p}$ denote the arc of the circle of radius $r$ in $F_{p}$ from $x$ to $y$ which contains $f(p)$. This arc has length $\pi r$ (see Figure 3 ).

Define the map $\Sigma(f)$ via

$$
\Sigma(f)(\{p\} \times[0,1])=\gamma_{p} .
$$

So, for example, $\Sigma(f)$ stretches each $\{p\} \times[0,1]$ by a factor of $\pi r$. It is not difficult to check that $\Sigma(f): S^{k+1} \rightarrow S(r)$ is an admissible map. Note also that $\Sigma(f)\left(S^{k+1}\right) \cap$ $(X \times\{0\})=f\left(S^{k}\right)$.

Suppose that $\widehat{\Sigma(f)}: B^{k+1} \rightarrow C(r)$ is an admissible filling of $\Sigma(f)$. Then $\widehat{\Sigma(f)} \cap$ $(X \times\{0\})$ gives an admissible filling of $f\left(S^{k}\right)$ in $X$, and this filling has volume at least $\delta^{k}(r)$, where $\delta^{k}(r)$ is the appropriate divergence function as in the definition of $\operatorname{div}_{k}\left(X^{n}\right)$.

Let a constant $\tau \ll 1$ be fixed. The reasoning above shows that for any $0 \leq \epsilon \leq \tau$, the map given by $\widehat{\Sigma(f)} \cap(X \times\{\epsilon\})$ gives an admissible filling of $\Sigma(f)\left(S^{k} \times\{\epsilon\}\right)$, and so this filling has volume at least $\delta^{k}(r-\epsilon)$. Since the leaves $\{X \times\{\epsilon\}\}_{0 \leq \epsilon \leq \tau}$ 
are parallel, we have

$$
\begin{aligned}
\operatorname{vol}(\widehat{\Sigma(f)}) & \geq \int_{0}^{\tau} \delta^{k}(r-\epsilon) d \epsilon \geq \int_{0}^{\tau} \delta^{k}(r-\tau) d \epsilon \\
& \geq \tau \cdot \delta^{k}(r-\tau) \sim \delta^{k}(r),
\end{aligned}
$$

as desired.

\section{Pulling-Off Spheres Along Flats}

In this section we give a polynomial upper bound for $\operatorname{div}_{k}\left(X \times \mathbf{R}^{k+1}\right)$ for any Hadamard manifold $X$ (Theorem 3.1).

Theorem 3.1 (a polynomial filling). Let $X$ be an Hadamard manifold. Then $\operatorname{div}_{k}\left(X \times \mathbf{R}^{k+1}\right)$ is a polynomial of degree at most $2 k+1$.

Proof. Let an $A$-admissible map $\gamma: S^{k} \rightarrow S(r)$ be given, where $S(r)$ is the sphere of radius $r$ around a chosen basepoint $x_{0} \in X \times \mathbf{R}^{k+1}$. Let $\pi: X \times \mathbf{R}^{k+1} \rightarrow \mathbf{R}^{k+1}$ be the natural projection.

Theorem 10.3.3 of $[\mathrm{E}+]$, which is an analogue of the deformation theorem of geometric measure theory, applied to $\pi \circ \gamma$ tells us that we may homotope $\gamma$ slightly (the homotopy being the identity in the $X$ factor) to a map $\gamma^{\prime}$ so that:

- $\pi \circ \gamma^{\prime}\left(S^{k}\right)$ lies outside the open unit ball in $\mathbf{R}^{k+1}$;

- There exists $C=C(k)>0$ so that $\operatorname{vol}_{k}\left(\gamma^{\prime}\left(S^{k}\right)\right) \leq C \cdot \operatorname{vol}_{k}\left(\gamma\left(S^{k}\right)\right)$;

- The volume of the homotopy is at most $C \cdot \operatorname{vol}_{k}\left(S^{k}\right)$.

Furthermore, one may check that adjoining the homotopy to an admissible filling of $\gamma^{\prime}$ gives an admissible filling of $\gamma$. So from now on we will assume that $\gamma$ satisfies the above properties.

We now give an admissible filling $\hat{\gamma}: B^{k+1} \rightarrow C(r)$ of $\gamma$. The filling is given by the tracks of $\gamma$ under a sequence of 4 homotopies which homotope $\gamma$ (outside $B(r)$ ) to a point; this filling is illustrated in Figure 4.

Here is the sequence of homotopies:

1. Radial projection in the $\mathbf{R}^{k+1}$ factor to the $3 r$-sphere in $\mathbf{R}^{k+1}:(x, \vec{v}) \mapsto$ $\left(x, t \cdot \frac{\vec{v}}{\|\vec{v}\|}\right), 1 \leq t \leq 3 r$. Since $\pi \circ \gamma$ lies outside the open unit ball in $\mathbf{R}^{k+1}$, this radial projection is well-defined, and increases the $k$-volume of $\gamma$ by at most a factor of $D r^{k}$ for some constant $D=D(k)$. Hence the $k$-volume of the image of $\gamma$ under this radial projection is at most $\left.D r^{k} \cdot \operatorname{vol}_{k}(\gamma) \leq D A r^{2 k}\right)$.

2. Coning-off in the $X$ factor: $(x, \vec{v}) \mapsto\left(\sigma_{x}(t), \vec{v}\right), 0 \leq t \leq 1$, where $\sigma_{x}$ is the (unique) geodesic in $X$ from $x$ to $x_{0}$.

3. Translating to the $5 r$-sphere in the $X$ factor: Let $x_{1}$ be a fixed point on the sphere of radius $5 r$ in $X$, and let $\tau$ be the unique geodesic from $x_{0}$ to $x_{1}$. Now perform $\left(x_{0}, \vec{v}\right) \mapsto(\tau(t), \vec{v}), 0 \leq t \leq 1$.

4. Coning-off in the $\mathbf{R}^{k+1}$ factor: $\left(x_{1}, \vec{v}\right) \mapsto\left(x_{1},(1-t) \cdot \vec{v}\right), 0 \leq t \leq 1$.

It is easy to check that the images of these homotopies lies outside $B(r)$, since the metric on $X \times \mathbf{R}^{k+1}$ is just the product metric. Piecing together these homotopies gives a map $\gamma_{2}: S^{k} \times[0,1] \rightarrow C(r)$ with the image $\gamma_{2}\left(S^{k} \times\{1\}\right)$ being the point $\left(x_{1}, \overrightarrow{0}\right)$. The map $\gamma_{2}$ induces a map on the cone on $S^{k}$, that is, a map $\hat{\gamma}: B^{k+1} \rightarrow$ $C(r)$. The map $\hat{\gamma}$ is easily seen to be an admissible filling of $(k+1)$-volume at most 
(by Fubini):

$$
\left(D A r^{2 k}\right) \cdot(3 r)+\left(D A r^{2 k}\right) \cdot r+\left(D A r^{2 k}\right) \cdot(5 r)+\left(D A r^{2 k}\right) \cdot(3 r) \leq 12 D A r^{2 k+1} .
$$

Hence $\operatorname{div}_{k}\left(X \times \mathbf{R}^{k+1}\right) \preceq r^{2 k+1}$.

$\mathrm{X}$

1.

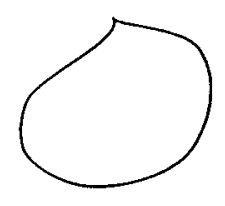

2.

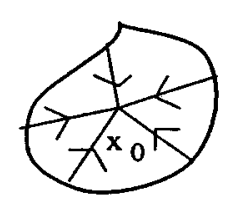

3.

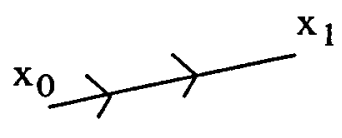

4.

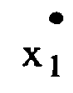

$\mathbf{R}^{\mathrm{k}+1}$
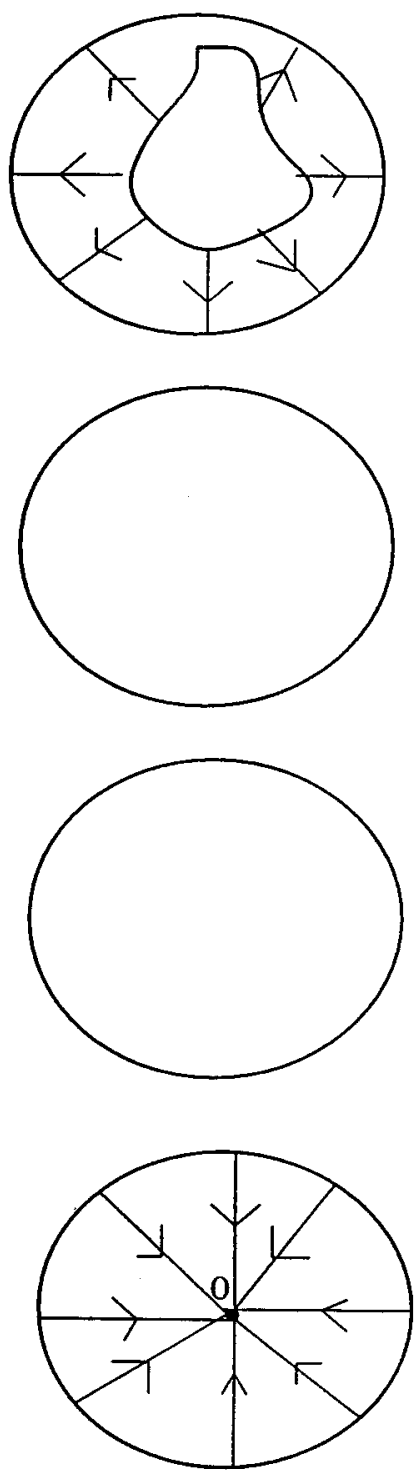

Figure 4. This figure gives a sequence of 4 homotopies which homotope $\gamma$ (outside $B(r)$ ) to a point. The homotopy is illustrated by its projections onto the $X$ and $\mathbf{R}^{k+1}$ factors. 


\section{Transverse Flats and Hyperbolic Spaces in Products}

It is a surprising fact, probably noted by Gromov, ${ }^{1}$ that there is, for example, a quasi-isometrically embedded copy of $\mathbf{H}^{3}$ inside $\mathbf{H}^{2} \times \mathbf{H}^{2}$ In the first part of this section, we show that there are quasi-isometric embeddings of hyperbolic spaces and solvable Lie groups in products of hyperbolic spaces, although these embeddings are not quasi-convex. We then apply the first embeddings to find a lower bound for certain $\operatorname{div}_{k}$ of products of hyperbolic spaces.

The idea is to exploit the fact that, in a product $X$ of hyperbolic spaces, there is a flat and a nicely embedded hyperbolic space whose dimensions add to $\operatorname{dim}(X)+1$. The flat is used to find a polynomial-volume sphere; the hyperbolic space is used to show that any admissible filling of this sphere has exponential volume.

Recall that a subset $Y$ of a metric space $X$ is called quasiconvex in $X$ if there is a constant $D \geq 0$ so that any geodesic in $X$ between points $y_{1}, y_{2} \in Y$ lies in the $D$-neighborhood of $Y$.

Proposition 4.1 (quasi-isometric embeddings). There are quasi-isometric embeddings of $\mathbf{H}^{\left(m_{1}+\cdots+m_{n}\right)-k+1}$ and of an $\left(\left(m_{1}+\cdots+m_{n}\right)-k+1\right)$-dimensional solvable Lie group in $X=\mathbf{H}^{m_{1}} \times \cdots \times \mathbf{H}^{m_{k}}$, where each $m_{i}>1$. These embeddings are not quasiconvex.

Examples. Proposition 4.1 shows that there are quasi-isometrically embedded (but not quasiconvex) copies of $\mathbf{H}^{3}$ and of the three-dimensional geometry Sol in $\mathbf{H}^{2} \times \mathbf{H}^{2}$, and that there are quasi-isometrically embedded copies of $\mathbf{H}^{4}$ in $\mathbf{H}^{2} \times \mathbf{H}^{2} \times \mathbf{H}^{2}$ and of $\mathbf{H}^{5}$ in $\mathbf{H}^{3} \times \mathbf{H}^{3}$.

Proof. Let $\gamma(t)=\left(\gamma_{1}(t), \ldots, \gamma_{k}(t)\right)$ be an infinite diagonal geodesic in $X$, parameterized by arc length, so that $\gamma_{i}$ traces out (at a speed of $1 / \sqrt{k}$ times unit speed) an infinite geodesic $g_{i}$ in $H^{m_{i}}$. For each $1 \leq i \leq k$, let $S_{i}^{+}(t)$ (respectively $S_{i}^{-}(t)$ ) denote the horosphere in $\mathbf{H}^{m_{i}}$ which is centered at $\gamma_{i}(+\infty)$ (respectively $\gamma_{i}(-\infty)$ ) and which contains the point $\gamma(t)$. When using the coordinates $\mathbf{R}^{n-1} \rtimes \mathbf{R}$ for hyperbolic space $\mathbf{H}^{n}$ (where $t \in \mathbf{R}$ acts on $\mathbf{R}^{n-1}$ by $\left(x_{1}, \ldots, x_{n}\right) \mapsto e^{-t}\left(x_{1}, \ldots, x_{n}\right)$ ) we shall refer to the $\mathbf{R}^{n-1}$ coordinates as horospherical and the $\mathbf{R}$ coordinate as vertical.

Now we define the quasi-isometrically embedded copy of hyperbolic space

$$
Y=\mathbf{H}^{\left(m_{1}+\cdots+m_{k}\right)-k+1}=\mathbf{R}^{\left(m_{1}+\cdots+m_{k}\right)-k} \rtimes \mathbf{R}
$$

in $\mathbf{H}^{m_{1}} \times \cdots \times \mathbf{H}^{m_{k}}$ to be the set of points

$$
\left\{\left(x_{1}, \ldots, x_{k}\right): x_{i} \in S_{i}^{+}(t), t \in \mathbf{R}\right\} .
$$

It is worth noting that this set contains the geodesic $\gamma$ which is the vertical $\mathbf{R}$ in $\mathbf{R}^{\left(m_{1}+\cdots+m_{k}\right)-k} \rtimes \mathbf{R}$. Each horosphere $S_{i}$ contributes a copy of $\mathbf{R}^{m_{i}-1}$ which is scaled by a factor of $e^{-t / \sqrt{k}}$. Hence the metric induced on $\mathbf{R}^{\left(m_{1}+\cdots+m_{k}\right)-k} \rtimes \mathbf{R}$ is given by

$$
d t^{2}+e^{-t / \sqrt{k}} d s^{2}
$$

where $d s^{2}$ denotes the Euclidean metric on $\mathbf{R}^{\left(m_{1}+\cdots+m_{k}\right)-k}$.

First we show that $Y \subset X$ is a quasi-isometric embedding. For any two points $p, q \in Y$ we have $d_{X}(p, q) \leq d_{Y}(p, q)$. For the rest of the proof we refer to Figure 5. Let $p_{i}$ and $q_{i}$ denote the projections of $p$ and $q$ onto the factors $\mathbf{H}^{m_{i}}$, and let $\gamma_{i}$

\footnotetext{
${ }^{1}$ We somehow remember that this fact was stated by Gromov somewhere in [Gr], but we've been unable to locate the exact reference.
} 


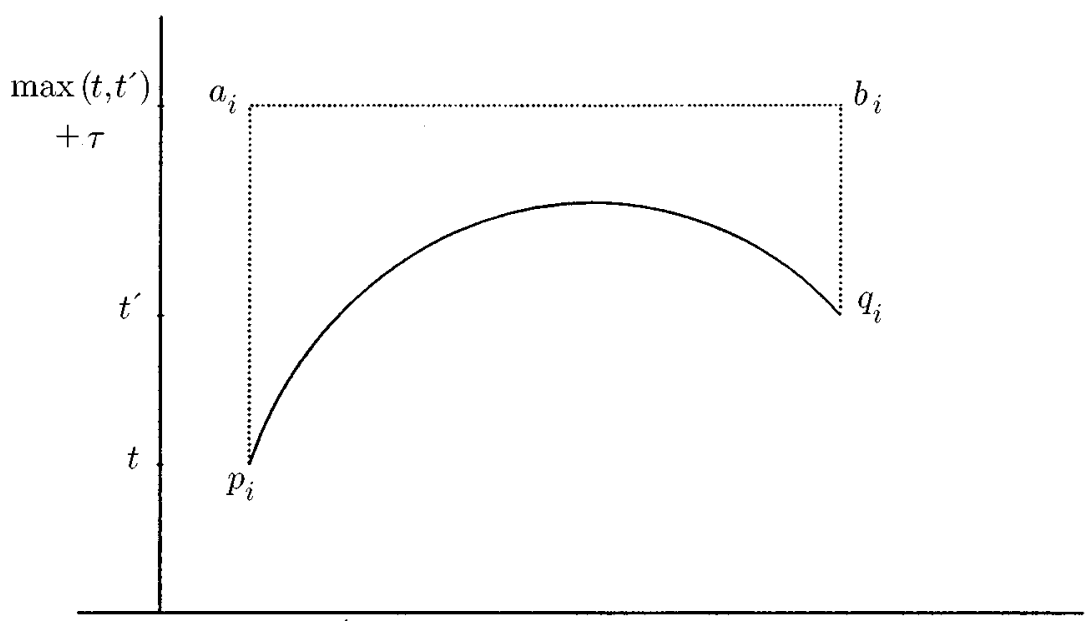

Figure 5. The hyperbolic space is quasi-isometrically embedded.

denote the geodesic in $\mathbf{H}^{m_{i}}$ between $p_{i}$ and $q_{i}$. Since $p, q \in Y$, the $p_{i}$ all have the same vertical coordinate $t$ and the $q_{i}$ all have the same vertical coordinate $t^{\prime}$.

For each $1 \leq i \leq k$ let

$$
\pi_{i}\left(x_{1}, \ldots, x_{m_{i}}, t\right)=t
$$

be the horospherical projection of $\mathbf{H}^{m_{i}}$ onto our original (vertical) geodesic $g_{i}$, and let

$$
\tau=\sup _{i} \operatorname{length}\left(\pi_{i}\left(\gamma_{i}\right)\right) .
$$

Denote by $a_{i}$ and $b_{i}$, respectively, the points in $\mathbf{H}^{m_{i}}$ which lie vertically above $p_{i}$ and $q_{i}$ on the horospherical level $\min \left\{t, t^{\prime}\right\}+\tau$.

Now consider the path in $Y$ from $p$ to $q$ which consists of the diagonal geodesic segments from $p=\left(p_{i}, \ldots, p_{k}\right)$ to $\left(a_{1}, \ldots, a_{k}\right)$ and from $\left(b_{1}, \ldots, b_{k}\right)$ to $q=$ $\left(q_{1}, \ldots, q_{k}\right)$, and a geodesic (in the intrinsic metric on the product of horospheres $\left.S_{1}^{+} \times \cdots \times S_{k}^{+}\right)$from $\left(a_{1}, \ldots, a_{k}\right)$ to $\left(b_{1}, \ldots, b_{k}\right)$. Each of the diagonal geodesics have length bounded by $\sqrt{k} \tau$, and the other geodesic segment has length with bound $2 \sqrt{k}$.

Thus the length of this path is less than $2 \sqrt{k} \tau+2 \sqrt{k}$, and so we have

$$
d_{Y}(p, q) \leq 2 \sqrt{k} \tau+2 \sqrt{k} \leq 2 \sqrt{k} d_{\mathbf{H}^{m_{j}}}\left(p_{j}, q_{j}\right)+2 \sqrt{k} \leq 2 \sqrt{k} d_{X}(p, q)+2 \sqrt{k},
$$

where $\mathbf{H}^{m_{j}}$ is the factor realizing the maximum of the lengths of $\pi_{i}\left(\gamma_{i}\right)$.

Now we show that $Y$ is not quasiconvex in $X$. Consider the two points $\left(p_{1}, p_{2}, \ldots, p_{k}\right)$ and $\left(q_{1}, p_{2}, \ldots, p_{k}\right)$ in $Y$, where $p_{1}$ and $q_{1}$ lie on the same horosphere in $\mathbf{H}^{m_{1}}$. The $X$ geodesic between these points is just the geodesic in $\mathbf{H}^{m_{1}}$ from $p_{1}$ to $q_{1}$ (with the other coordinates just constant at the point $\left(p_{2}, \ldots, p_{k}\right)$ ). Clearly this does not lie in $Y$; in fact, the distance from its midpoint $\left(r_{1}, p_{2}, \ldots, p_{k}\right)$ to $Y$ is given by

$$
\min _{l_{1}+l_{2}=l} \sqrt{(k-1) l_{1}^{2}+l_{2}^{2}},
$$

where $l$ is the vertical height of the geodesic between $p_{1}$ and $q_{1}$ in $\mathbf{H}^{m_{1}}$. This can be made arbitrarily large by choosing $p_{1}$ and $q_{1}$ far apart. 
Finally, note that there are quasi-isometrically embedded copies of solvable Lie groups in $X$; these are just the horospheres in $X$. More explicitly, for example, define $Z$ in $X$ to be the set of points

$$
Z=\left\{\left(x_{1}, \ldots, x_{k}\right): x_{1} \in S_{1}^{+}(t), x_{i} \in S_{i}^{-}(t)(i=2, \ldots, k), t \in \mathbf{R}\right\} .
$$

The reader can verify that the induced metric on $Z=\mathbf{R}^{\left(m_{1}+\cdots+m_{k}\right)-k} \rtimes \mathbf{R}$ is given by

$$
d t^{2}+e^{-t / \sqrt{k}} d s_{1}^{2}+e^{t / \sqrt{k}} d s_{2}^{2}
$$

where $d s_{1}^{2}$ denotes the Euclidean metric on $\mathbf{R}^{m_{1}-1}$ and $d s_{2}^{2}$ denotes the Euclidean metric on $\mathbf{R}^{\left(m_{2}+\cdots+m_{k}\right)-k+1}$, and that $Z$ is quasi-isometrically embedded in $X$.

The geodesic $\gamma$ of Proposition 4.1 is contained in the $k$-flat $F=\gamma_{1} \times \cdots \times \gamma_{k}$. This $k$-flat is foliated by parallel geodesics of the form

$$
\gamma_{\left(s_{1}, \ldots, s_{k}\right)}(t)=\left(\gamma_{1}\left(t+s_{1}\right), \ldots, \gamma_{k}\left(t+s_{k}\right)\right)
$$

where $s_{1}+\cdots+s_{k}=0$. The corresponding family of hyperbolic spaces

$$
Y_{\left(s_{1}, \ldots, s_{k}\right)}=\left\{\left(x_{1}, \ldots, x_{k}\right): x_{i} \in S_{i}^{+}\left(t+s_{i}\right), t \in \mathbf{R}\right\}
$$

gives a codimension $(k-1)$ foliation of $X$, where each $Y_{\left(s_{i}, \ldots, s_{k}\right)}$ intersects the $k$-flat $\gamma_{1} \times \cdots \times \gamma_{k}$ in the geodesic $\gamma_{\left(s_{1}, \ldots, s_{k}\right)}$.

Lemma 4.2. For any points $p \in Y_{\left(s_{1}, \ldots, s_{k}\right)}$ and $q \in Y_{\left(s_{1}^{\prime}, \ldots, s_{k}^{\prime}\right)}$ we have

$$
d_{X}(p, q) \geq \sqrt{\left(s_{1}-s_{1}^{\prime}\right)^{2}+\cdots+\left(s_{k}-s_{k}^{\prime}\right)^{2}} .
$$

Proof. Perpendicular projection of $X$ onto the $k$-flat $\gamma_{1} \times \cdots \times \gamma_{k}$ is a distance nonincreasing map which takes $p$ and $q$ to points on the geodesic lines $\gamma_{\left(s_{1}, \ldots, s_{k}\right)}$ and $\gamma_{\left(s_{1}^{\prime}, \ldots, s_{k}^{\prime}\right)}$ respectively. The $X$-distance between the image points is the same as the distance in the $k$-flat, which is bounded below by $\sqrt{\left(s_{1}-s_{1}^{\prime}\right)^{2}+\cdots+\left(s_{k}-s_{k}^{\prime}\right)^{2}}$.

Theorem 4.3 (some hard-to-fill spheres). Let $X=\mathbf{H}^{m_{1}} \times \cdots \times \mathbf{H}^{m_{k}}$, each $m_{i}>1$, be a product of $k$ hyperbolic spaces. Then $\operatorname{div}_{k-1}(X)$ is exponential.

Proof. Consider the family of hyperbolic spaces $\left\{Y_{s}\right\}$ as above, where $s \in B(1)$, the ball of radius 1 in $F$. Recall that $F=\gamma_{1} \times \cdots \times \gamma_{k}$ is a totally geodesic, isometrically embedded copy of $\mathbf{R}^{k}$ in $X$. Also recall that the volume of a sphere of radius $r$ in $\mathbf{R}^{k}$ is $a r^{k}$ for some constant $a$ depending only on $k$.

Now let $A>a$ and $r>0$ be given, and let $T=F \cap S(r)$. Then $T$ is a $(k-1)$ dimensional sphere lying on the sphere $S(r)$ of radius $r$ about the origin in $X$. Since $T \subset F$ and $F$ is a flat in $X$, we have $\operatorname{vol}_{k-1}(T) \leq a r^{k} \leq A r^{k}$. We claim that any filling of $T$ outside of $S(r)$ has $(k+1)$-volume on the order of $e^{r}$.

To prove this claim, suppose that $\widehat{T}$ is an admissible filling of $T$. First note that the intersection $T \cap Y_{s}$ in $X$ has dimension 0 ; in fact, $T \cap Y_{s}$ consists precisely of two points, $T \cap \gamma_{s}=\left\{x_{s}, y_{s}\right\}$. Since $x_{s}$ and $y_{s}$ are each within a distance of 1 from antipodal points of $S(R)$ lying on the geodesic $\gamma_{0}$, it follows that $x_{s}$ and $y_{s}$ are each within a distance of 1 from antipodal points on the sphere $S^{\prime}(r)$ of radius $r$ in $Y$. Now $\widehat{T} \cap Y_{s}$ is a one-dimensional arc in $Y_{s}$ which connects $x_{s}$ to $y_{s}$ outside of $S^{\prime}(r)$. Since $Y_{s}$ is a hyperbolic space, $\operatorname{div}_{0}\left(Y_{s}\right)=\exp$, so that the arc $\widehat{T} \cap Y_{s}$ has length at least $C e^{r}$ for some constant $C$ which is independent of $r$. We note that since $s \in B(1)$, the constant $C$ may be chosen to work for all $Y_{s}$. 
Now

$$
\begin{array}{rlrl}
\operatorname{vol}_{k+1}(\widehat{T}) & =\int_{B(1)} \operatorname{vol}_{k+1}\left(\widehat{T} \cap Y_{s}\right) d \mu(s) & \\
& \geq \int_{B(1)} C e^{r} d \mu(s) & & \text { since }\left|\widehat{T} \cap Y_{s}\right| \geq C e^{r} \\
& \geq C e^{r} \mu(B(1)) & & \text { by Lemma } 4.2,
\end{array}
$$

and we are done.

Remark. The reason it is necessary to use Lemma 4.2 is that it is possible to have, for example, a disc foliated by an interval's worth of lines of length $e^{r}$, but with the area of the disc being constant. For example, consider a long, thin quadrilateral in the hyperbolic plane: it is foliated by lines of length $e^{r}$ for $r$ large, but its area is bounded by a universal constant; the reason is that the leaves of the foliation are bent so that they come very close together, on the order of $e^{-r}$, in fact.

\section{An Example and Some Questions}

As stated above, we view the invariants $\operatorname{div}_{k}(X)$ as basic geometric quantities to be computed. In particular, computations of $\operatorname{div}_{k}(X)$ provide a good test of one's understanding of the geometry of $X$. Finally, we believe that the invariants $\operatorname{div}_{k}\left(X^{n}\right)$ are computable for many more examples than are covered in this paper.

Question. Compute $\operatorname{div}_{k}(X)$ for symmetric spaces $X$ of noncompact type. The simplest case not covered in this paper is $\operatorname{div}_{2}\left(\mathbf{H}^{2} \times \mathbf{H}^{2}\right)$. We believe that $\operatorname{div}_{1}(X) \sim$ $e^{r}$ for the symmetric space $X=S L_{n}(\mathbf{R}) / S O_{n}(\mathbf{R})$. In [EF], it is shown that there is a foliation of symmetric spaces $X$ by 'hyperbolic-like' spaces. Each subspace is (a translate of) a $K$-orbit of an infinite ray in the positive Weyl chamber in $A$. Here $A$ and $K$ come from the standard KAN decomposition of $\operatorname{Isom}(X)$. Is it possible to use this observation together with the same trick as in the proof of Theorem 4.3 to compute $\operatorname{div}_{k}$ for symmetric spaces?

In $[\mathrm{KL}]$ (see also $[\mathrm{EF}]$ ) it is shown, among other things, that two noncompact symmetric spaces are quasi-isometric if and only if they are isometric. It would be nice if there were a list of numerical invariants to distinguish these spaces. The computations of the invariants $\operatorname{div}_{k}$ above can be used to distinguish some simple cases.

Example. If $\mathbf{H}^{a} \times \mathbf{R}^{b}$ is quasi-isometric to $\mathbf{H}^{n} \times \mathbf{R}^{m}$, then $a=n$ and $b=m$. Since virtual cohomological dimension is a quasi-isometry invariant for groups with finite classifying space (see [BW, Ge2]), and since both spaces are contractible and admit a cocompact discrete group action, we know that $a+b=n+m$.

Suppose $m>b$ (the case $b>m$ is similar). Iterating Theorem $2.1 b$ times gives $\operatorname{div}_{b}\left(\mathbf{H}^{a} \times \mathbf{R}^{b}\right) \succeq \operatorname{div}_{0}\left(\mathbf{H}^{a}\right)$, which is exponential. But by Theorem 3.1 we know that $\operatorname{div}_{b}\left(\mathbf{H}^{n} \times \mathbf{R}^{m}\right)$ is polynomial since $m>b$, a contradiction. So $m=b$ and $n=a$.

Question. Which symmetric spaces can be distinguished by the invariants $\operatorname{div}_{k}$ ? Can the $\operatorname{div}_{k}(X)$ be used to detect the rank of a noncompact symmetric space $X$ ? 


\section{ApPendix A}

In this appendix we give a technical proposition which was needed to show that the $\operatorname{div}_{k}\left(X^{n}\right)$ are quasi-isometry invariants. This proposition is well-known (see, e.g., $[\mathrm{BW}]$ ), but as we couldn't find a detailed proof in print, we include one here.

Proposition A.1 (connect-the-dots). Suppose $f: X \rightarrow Y$ is a quasi-isometry between Hadamard manifolds, and suppose that $X$ admits a cocompact lattice. Then $f$ is a bounded distance from a (continuous) lipschitz map $f^{\prime}: X \rightarrow Y$; that is, $\sup _{x \in X} d_{Y}\left(f(x), f^{\prime}(x)\right) \leq C$ for some constant $C>0$.

Proof. Since $X$ admits some compact quotient $M=X / \Gamma$, it is possible to lift a triangulation of $M$ to to a $\Gamma$-equivariant triangulation of $X$. Note that there are finitely many isometry types of simplices in this triangulation of $X$.

The map $f^{\prime}$ is defined inductively on the skeleta of the triangulation. On vertices we simply define $f^{\prime}$ to equal $f$. Suppose $f^{\prime}$ is defined on the $k$-skeleton of the triangulation; then for each $(k+1)$-simplex $\sigma$, we have a lipschitz map defined on $\partial(\sigma)$, which is a sphere. This map extends to a lipschitz map on $\sigma$ by Whitney's Extension Theorem. Do this for each different $(k+1)$-simplex $\sigma$; the point is that there are only finitely many different lipschitz constants since there are only finitely many isometry types of simplices; hence the map $f^{\prime}$ is lipschitz with constant the maximum of the Whitney lipschitz constants on the finitely many isometry types of simplices.

\section{REFERENCES}

[BW] J. Block and S. Weinberger, Large scale homology theories and geometry, Geometric Topology (Athens, GA, 1993), AMS/IP Stud. Adv. Math., vol. 2, part 1, Amer. Math. Soc., Providence, RI, 1997, pp. 522-569. CMP 98:01

[E+] D.B.A. Epstein, J. Cannon, D.F. Holt, S. Levy, M.S. Paterson and W.P. Thurston, Word Processing in Groups, Jones and Bartlett, Boston, MA, 1992. MR 93i:20036

[EF] A. Eskin and B. Farb, Quasi-flats and rigidity in higher rank symmetric spaces, J. Amer. Math. Soc. 10 (1997), 653-692. CMP 97:11

[Ge1] S.M. Gersten, Quadratic divergence of geodesics in $C A T(0)$ spaces, Geometric and Functional Analysis, 4 (1994), 37-51. MR 95h:53057

[Ge2] S. Gersten, Quasi-isometry invariance of cohomological dimension, Comptes Rendues Acad. Sci. Paris, Ser. I Math. 316 (1993), 411-416. MR 94b:20042

[Gr] M. Gromov, Asymptotic Invariants of Infinite Groups, Geometric Group Theory, G. Niblo and M. Roller, eds., LMS Lecture Notes 182, Cambridge Univ. Press, 1993, pp. 1-295. MR 95m:20041

[KL] B. Kleiner and B. Leeb, Rigidity of quasi-isometries for symmetric spaces of higher rank, to appear in Publ. Math. IHES.

Department of Mathematics, Cornell University, Ithaca, New York 14853

E-mail address: brady@math.cornell.edu

Department of Mathematics, University of Chicago, 5734 University Ave., Chicago, ILLINOIS 60637

E-mail address: farb@math.uchicago.edu 